\title{
GASTROPLASTIA A COLLIS ASSOCIADA À FUNDOPLICATURA PARCIAL OU TOTAL: ESTUDO EXPERIMENTAL
}

\author{
Collis gastroplasty associated to parcial or total fundoplication: experimental study
}

Luiz Roberto LOPES, Nelson Adami ANDREOLLO

Trabalho realizado na Disciplina de Moléstias do Aparelho Digestivo - Departamento de Cirurgia - Faculdade de Ciências Médicas - Universidade Estadual de Campinas. Campinas, SP, Brasil.

DESCRITORES - Esofagite péptica. Estenose esofágica. Modelos animais.

\section{Correspondência: \\ Luiz Roberto Lopes, \\ e-mail: lopesIr@fcm.unicamp.br}

Fonte de financiamento: não há

Conflito de interesses: não há

Recebido para publicação: 15/11/2010

Aceito para publicação: 21/12/2010

HEADINGS - Esophagitis, peptic. Esophageal stenosis. Models, animal.
RESUMO - Racional - A esofagite de refluxo complicada com estenose e encurtamento do órgão é doença de difícil tratamento cirúrgico com frequente insucesso. A gastroplastia a Collis associada à fundoplicatura parcial ou total pode ser empregada nesta situação. Objetivo - Avaliar a técnica da gastroplastia a Collis com fundoplicatura parcial ou total em cães. Métodos - Sete cães foram submetidos a Collis-Lind (Grupo A) e quatro a Collis-Nissen (Grupo B) após miotomia da junção esofagogástrica. O refluxo foi avaliado com medidas de $\mathrm{pH}$ e as pressões na transição esofagogástrica com microtransdutores. Resultados Após a miotomia ocorreu refluxo livremente em todos os animais. Entretanto, após a operação de Collis-Lind ou Collis-Nissen o refluxo espontâneo não ocorreu. A aplicação de pressão manual sobre o estômago, promoveu refluxo em 28,5 \% dos animais do Grupo A. A avaliação das pressões na peça cirúrgica mostrou que não houve diferença na zona de alta pressão, nos dois grupos. Conclusão - A operação de Collis-Lind ou Collis-Nissen impediu o refluxo gastroesofágico. As medidas das pressões e a extensão da zona de alta pressão foram semelhantes nos dois grupos.

ABSTRACT - Background - Reflux esophagitis complicated by stenosis and shortening of the organ is a difficult disease to surgical treatment with frequent failure. The Collis gastroplasty associated with a partial or total fundoplication can be employed in this situation. Objective - To evaluate the technique of fundoplication with partial or total Collis gastroplasty in dogs. Methods - Seven dogs underwent a Collis-Lind (Group A) and four to Collis-Nissen (Group B) after myotomy of the gastroesophageal junction; reflux was evaluated with measurements of $\mathrm{pH}$ and pressures in the esophagogastric transition with microtransducer. Results - After myotomy reflux occurred freely in all animals. However, after Collis-Lind or CollisNissen procedures, it not ocurred spontaneously. The application of manual pressure on the stomach caused reflux in $28.5 \%$ of animals in Group A. The assessment of pressures in the surgical specimen showed no difference in the area of high pressure in both groups. Conclusion - The Collis-Lind and Collis-Nissen procedures prevent gastroesophageal reflux. The pressure measurements and the extension of the high pressure zone were similar in both groups.

\section{INTRODUÇÃO}

A esofagite de refluxo é doença caracterizada por inflamação da mucosa esofagiana causada por repetidos e prolongados contatos do conteúdo gastroduodenal refluído para o esôfago ${ }^{3}$. Além do problema do estreitamento da luz do órgão, outra situação que pode acompanhar as alterações inflamatórias transmurais, é o esôfago curto adquirido e motivo de controvérsia no seu diagnóstico ${ }^{10,26}$. Mercer e Hill $(1986)^{19}$, acreditavam que sempre é possível obter segmento abdominal de esôfago para um reparo cirúrgico adequado, enquanto que outros autores reconhecem essa situação e enfatizam a influência negativa nos resultados cirúrgicos ${ }^{10,30}$. Em avaliação intraoperatória em oito diferentes centros de estudos, chegou-se a cerca de $20 \%$ a incidência de esôfago curto adquirido ${ }^{17}$.

O tratamento cirúrgico ideal visa restaurar o trânsito alimentar normal 
e prevenir o refluxo subsequente ${ }^{30}$. Com a aplicação das técnicas antirefluxo das fundoplicaturas na vigência de esôfago encurtado, a ocorrência de falha no tratamento é maior, pois a junção esofagogástrica (JEG) fica no abdome sob tensão, prejudicando a ação do esfíncter inferior do esôfago (EIE), além de poder ocorrer o escorregamento da JEG para o tórax, ficando o envolvimento da válvula na parte proximal gástrica, não sendo efetivo no controle do refluxo $0^{1,7,26,34}$.

Collis em $1957^{5}$ descreveu a operação da gastroplastia, na qual um alongamento do esôfago com a pequena curvatura gástrica era obtido e que tinha o mesmo calibre do esôfago sendo sua continuidade, alcançando um dos princípios da cirurgia antirefluxo que é obter segmento abdominal de esôfago mantendo o ângulo de entrada do esôfago no estômago, mas que se mostrou insuficiente, pois encontrou recorrência de sintomas ${ }^{6}$. Pearson, et al. ${ }^{23}$, associaram à gastroplastia uma fundoplicatura parcial tipo Belsey e Henderson ${ }^{12}$ preferiu associar a fundoplicatura total tipo Nissen, obtendo melhores resultados no controle do refluxo.

A técnica descrita por Collis associada à fundoplicatura total para tratamento dos pacientes portadores de esofagites e estenoses que levam a encurtamento do esôfago alcançava os principais objetivos propostos hoje: há preservação do esôfago, o esfíncter inferior não é excisado, os nervos vagos permanecem íntegros ${ }^{24}$, obtém-se um segmento de neoesôfago subdiafragmático para adequado reparo ${ }^{2,11}$, elimina-se a tensão no reparo ${ }^{2,23}$, utilizase tecido não inflamado para a fundoplicatura ${ }^{2,21,22}$, e mantém-se a angulação da JEG ${ }^{18}$. Está associada à baixa morbidade e mortalidade e baixa recorrência anatômica de hérnia e refluxo ${ }^{27}$.

A operação, a princípio, foi usada para doentes com estenose péptica do esôfago distal e consequente esôfago encurtado, porém atualmente, tem a sua indicação ampliada para reoperações de doentes com recidiva de refluxo, ou com outros fatores predisponentes ao refluxo ${ }^{25}$, tais como esofagite de refluxo grave com ou sem estenose, doença pulmonar obstrutiva crônica, obesidade, necessidade de esofagomiotomia, esclerodermia e grandes hérnias encarceradas ${ }^{21}$. Todos estes fatores citados interferem nas suturas usadas nas operações comuns e resultam em aumento de tensão no reparo, prejudicando a correção do refluxo².

A partir do advento da cirurgia por vídeo passou a ser empregada também por esta via sendo considerada a primeira escolha para os casos de esôfago encurtado grave e com grande dificuldade para solucionar o refluxo ${ }^{31}$.

Considerando-se difícil o tratamento das esofagites complicadas com estenose e encurtamento do esôfago e a pouca experiência com a técnica do alongamento tubular do esôfago com a pequena curvatura gástrica (técnica de Collis) associada à fundoplicatura parcial ou total, planejou-se um estudo experimental em cães com a finalidade de testar a técnica na prevenção do refluxo.

\section{MÉTODOS}

O trabalho foi aprovado pelo Comitê de Ética e Pesquisa Animal do Instituto de Biologia da Unicamp com o Protocolo n 1867-1.

Foram utilizados 11 cães com peso médio de 10,6 $\mathrm{kg}$ operados sob anestesia geral com pentobarbital sódico e mantidos em respiração positiva intermitente por tubação oro-traqueal com aparelho Takaoka.

A operação iniciou-se por laparotomia mediana, dissecção da JEG e isolamento do esôfago com dreno laminar fino. Para a ocorrência de refluxo, uma miotomia da JEG foi realizada em extensão de $4 \mathrm{~cm}$ na parede anterior do esôfago $(3 \mathrm{~cm})$ e do estômago (1 $\mathrm{cm}$ ) (Figura 1). Foi realizada a amarradura do duodeno para impedir o escape da solução de soro fisiológico $0,9 \%(500 \mathrm{ml})$ e $1,2 \mathrm{ml}$ de ácido clorídrico $12 \mathrm{~N}$, solução ajustada para atingir $\mathrm{pH} 3,0$, confirmado por pHmetro Beckman, injetada no estômago através de uma gastrostomia com sonda uretral $n^{\circ} 8$. Após isso, uma sonda Foley $\mathrm{n}^{\circ} 12$ era colocada pela boca do animal até o terço distal do esôfago para a coleta de líquido refluído para análise do seu $\mathrm{pH}$ através da leitura em fita do indicador universal de $\mathrm{pH}-0-14$ Merck.

Com a comprovação da existência do refluxo após a miotomia longitudinal, a operação prosseguia com a realização do alongamento do esôfago em uma extensão de $4 \mathrm{~cm}$ a partir do fundo gástrico paralelo ao esôfago, com uma sonda Portex $n^{\circ} 8,5$ french colocada no esôfago para evitar o estreitamento do neoesôfago a ser criado (Figura 2).

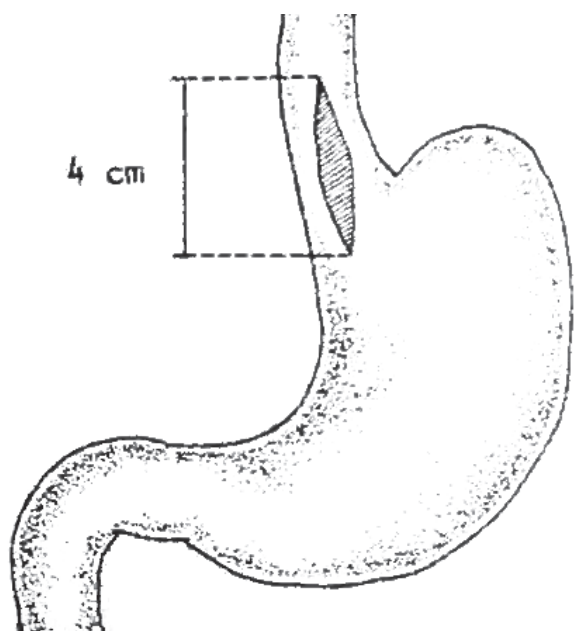

FIGURA 1 - Miotomia da transição esofagogástrica

Os animais foram divididos em dois grupos: Grupo A - em que a fundoplicatura realizada foi de $270^{\circ}$ à maneira de Lind (sete animais - Figura 3) e Grupo B - em que a fundoplicatura realizada foi de $360^{\circ}$ à maneira de Nissen (quatro animais - Figura 4). 


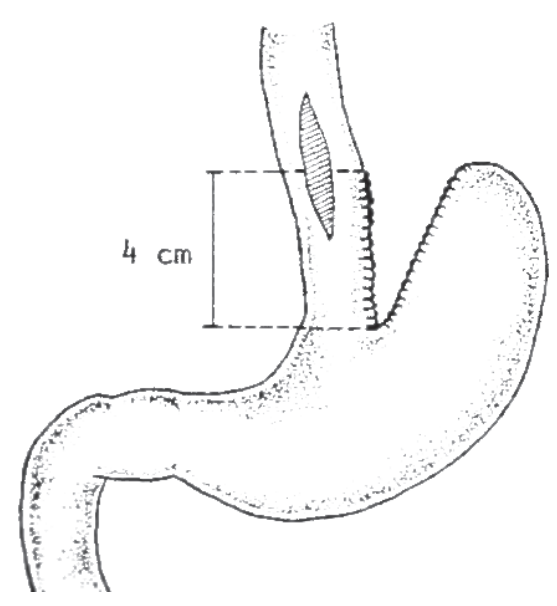

FIGURA 2 - Alongamento a Collis

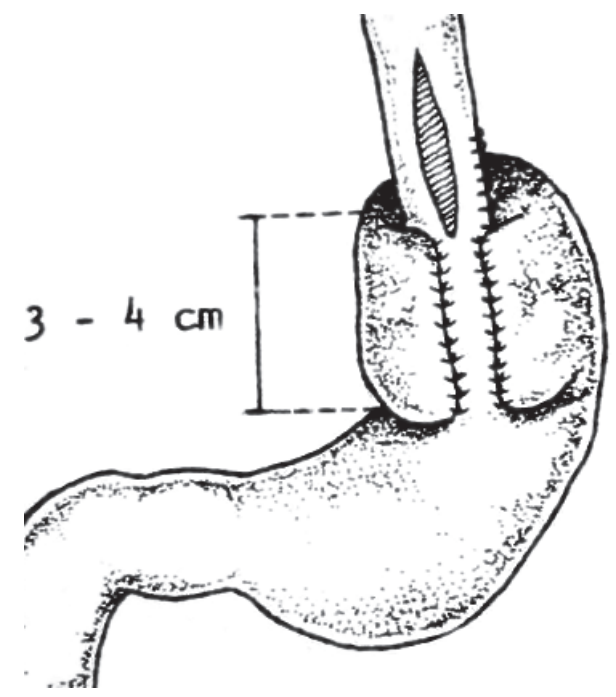

FIGURA 3 - Collis-Lind

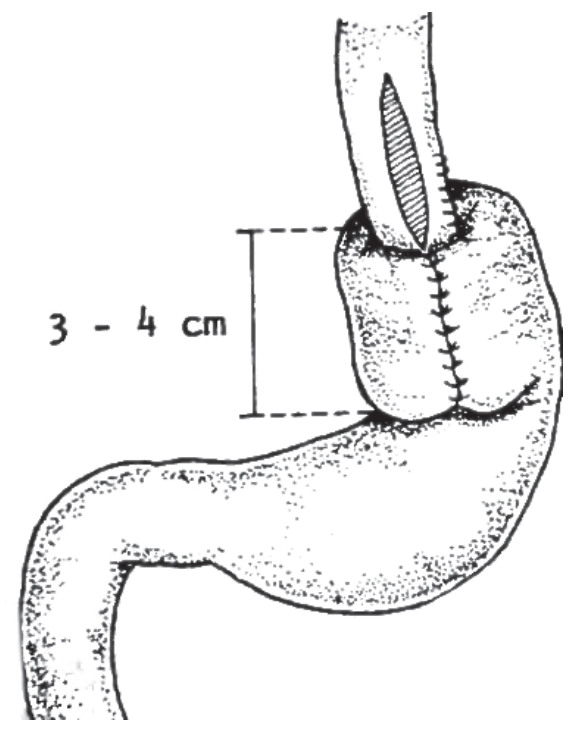

FIGURA 4 - Collis-Nissen

Após a realização da gastroplastia e da fundoplicatura, novamente era colocada a solução de pH ácido no estômago e observada a ocorrência de refluxo espontâneo e forçado aplicando-se compressão manual no estômago e coletando-se possível líquido refluído no esôfago. Previamente, o esôfago era lavado com soro fisiológico para não incorrer em erro de leitura do $\mathrm{pH}$ anteriormente ácido documentado, confirmado em leitura de fita do indicador universal de $\mathrm{pH}$.

Enquanto era infundida a solução ácida no estômago, media-se o volume injetado e anotava-se o valor máximo. Ocorrendo ou não o refluxo espontâneo, empregava-se posteriormente pressão manual na parede gástrica medindo-se a pressão intragástrica em $\mathrm{cm}$ de água através da sonda de gastrostomia conectada à uma coluna de água, observando a ocorrência do refluxo pela sonda Foley colocada no esôfago distal e medindo o seu pH.

Após os testes de refluxo ácido terem sido feitos e anotados os resultados, a peça cirúrgica era retirada constando do esôfago distal, o neo-esôfago confeccionado, o estômago e o duodeno abaixo da ligadura do mesmo.

A perveabilidade do neoesôfago era testada através da passagem do indicador do examinador e da sonda portex 8,5 french para comprovar a existência de estreitamento ou não.

O conjunto retirado era levado ao laboratório para estudo das pressões nesse neoesôfago criado cirurgicamente, por meio da colocação de cateter com microtransdutores de pressão sendo um deles intragástrico e o outro na área do alongamento e da fundoplicatura. Para este teste de pressão foi injetado soro fisiológico no estômago através da sonda de gastrostomia e medida as alterações de pressão nos dois pontos apontados. Também a aplicação de pressão manual sobre a parede anterior do estômago foi realizada e as alterações de pressão foram registradas em papel milimetrado próprio para esse exame. A retirada lenta e progressiva do cateter de microtransdutor foi realizada medindo-se a extensão da zona de alta pressão obtida com o alongamento e a fundoplicatura.

A análise estatística foi realizada dos parâmetros estudados com o teste T-Student e o teste de Wilcoxon considerando-se diferença significativa $p<0,05$.

\section{RESULTADOS}

Ocorreu refluxo espontâneo em todos os cães após a miotomia longitudinal da JEG com média de $171,42 \mathrm{ml}$ no grupo $\mathrm{A}$ e de $187,5 \mathrm{ml}$ no grupo $\mathrm{B}$ de líquido infundido $(p>0,05)$.

Após a realização do alongamento do esôfago e da confecção da fundoplicatura parcial ou total e infusão de líquido no estômago, não ocorreu refluxo espontâneo para o esôfago, com uma média de 371,42 $\mathrm{ml}$ no grupo A e de $425 \mathrm{ml}$ no grupo $B$ de volume infundido $(p>0,05)$.

A diferença entre o volume infundido para os 
grupos com a miotomia e após a realização da operação apresentou diferença significativa entre eles $(p<0,05)$, mostrando que a miotomia é suficiente para provocar refluxo gastroesofágico (grupo A - 171,42 ml/371,42 ml e grupo $B-187,5 \mathrm{ml} / 425 \mathrm{ml}$ ).

Com a aplicação de pressão manual sobre a parede gástrica, ocorreu refluxo em dois cães do grupo A (28,5\%) e em nenhum do grupo $B$ com $p>0,05$. As médias das pressões intragástricas obtidas nos dois grupos não mostraram diferenças significativas - $p>0,05$-(grupo A $27,14 \mathrm{cmH}_{2} \mathrm{O}$ e grupo $\mathrm{B}-30 \mathrm{~cm} \mathrm{H}_{2} \mathrm{O}$ ).

$\mathrm{O}$ estudo das pressões com microtransdutores da peça cirúrgica que foi ressecada no grupo A mostrou a média de pressão com infusão de soro fisiológico no estômago de $20 \mathrm{mmHg}$ e na gastroplastia de 15 $\mathrm{mmHg}$, enquanto que no grupo $B$ a média no estômago foi de $15,66 \mathrm{mmHg}$ e na gastroplastia de $14,66 \mathrm{mmHg}$ com $p>0,05$ em ambos os grupos. Na comparação das médias das pressões tanto no estômago como na gastroplastia entre os dois grupos não houve diferença significativa entre eles $(p>0,05)$.

Quando se aplicou pressão manual na parede gástrica e avaliaram-se as alterações de pressão no estômago e na gastroplastia de ambos os grupos, encontrou-se os seguintes valores: grupo A - 23,75 $\mathrm{mmHg}$ e $15,75 \mathrm{mmHg}$ e grupo $B-9,33 \mathrm{mmHg}$ e 12,66 $\mathrm{mmHg}$, havendo diferença significativa apenas na comparação entre a pressão obtida intragástrica no grupo A e o B.

A medida da extensão da zona de alta pressão obtida com a fundoplicatura no grupo $A$ foi de 3,25 $\mathrm{cm}$ e no grupo $B$ de $3,66 \mathrm{~cm}$, não sendo diferença significativa $(p>0,05)$.

\section{DISCUSSÃO}

O trabalho experimental conforme delineado, proporcionou através da realização da miotomia de 4 $\mathrm{cm}$ na parede anterior da JEG, o refluxo esperado em todos os animais para então avaliar a eficácia da técnica cirúrgica proposta.

As operações empregadas para a contenção do refluxo tentam restaurar a competência do EIE colocando-o em porção intra-abdominal, além de corrigir os possíveis defeitos da região do hiato ${ }^{3,20}$. Quando da existência de processo inflamatório intenso e esôfago encurtado, se a operação é realizada com a confecção de uma válvula anti-refluxo em um segmento curto ou inexistente de esôfago abdominal, essa fundoplicatura fica sob tensão e a tendência é a recorrência anatômica da hérnia hiatal e da persistência do refluxo ${ }^{26}$.

A realização de um tubo com a pequena curvatura gástrica como o alongamento do esôfago proposto por Collis em 1957 foi bem aceito ${ }^{6}$. O tubo tinha cerca de 7 a $9 \mathrm{~cm}$ de extensão com a aproximação do estômago ao tubo sem uma fundoplicatura significativa. Com isso conseguia-se alongamento funcional do esôfago distal, mantendo um ângulo agudo de entrada entre o esôfago e o estômago ${ }^{4,6}$.

Pearson, et al. ${ }^{23}$, associaram ao alongamento do esôfago uma fundoplicatura de $240^{\circ}$. Consideravam que não apenas o ângulo de entrada do esôfago ao estômago e o segmento de esôfago abdominal eram importantes, mas que, ao agregar uma fundoplicatura ao redor do neoesôfago, criava-se um mecanismo valvular. Com isso utilizava-se uma região livre de processo inflamatório, mais fácil manipulação, menor risco de lesão e de insucesso a longo prazo.

Os benefícios desta técnica empregada alcançavam um segmento de zona de alta pressão intra-abdominal, o EIE era preservado e não excisado ou destruído, os nervos vagos mantidos íntegros com ação da pressão positiva abdominal sobre essa região ${ }^{7,24}$.

Henderson $^{12}$ associou à gastroplastia uma fundoplicatura total à maneira de Nissen, pois em trabalho do seu grupo com a operação de Collis-Belsey encontrou $44,6 \%$ de refluxo radiológico e de $25,7 \%$ de sintomas importantes, apesar de apenas $1,5 \%$ de recorrência de hérnia hiatal.

Steichen ${ }^{29}$ descreveu a operação de Collis-Nissen em 77 pacientes onde encontrou 1,2\% de recorrência de hérnia hiatal e nenhum caso de refluxo radiológico ou sintomático.

Thomas-Ridocci, et al. ${ }^{33}$, comparando a operação de Nissen com a de Collis-Nissen, encontraram melhor resultado com a segunda com $6,6 \%$ e $0 \%$ de sintomas de recorrência e hérnia hiatal recidivada em $18 \%$ e $0 \%$ respectivamente. Já a manometria foi semelhante nos dois grupos e a pHmetria mostrou refluxo em $10 \%$ e $5 \%$ respectivamente, concluindo que a operação da gastroplastia com fundoplicatura foi melhor, apesar destes doentes serem mais graves que os do outro grupo.

No presente trabalho optou-se por realizar 0 estudo do alongamento do esôfago em uma extensão de $4 \mathrm{~cm}$ e de associar fundoplicatura em uma extensão igual recobrindo praticamente todo o tubo confeccionado.

A comparação dos dois tipos de fundoplicatura (parcial ou total) foi realizada, pois, em determinadas situações é necessário empregar a fundoplicatura parcial. Em trabalho experimental em gatos comparando a operação de Collis-Belsey e Collis-Nissen, ficou mostrado ser claramente superior a fundoplicatura total na contenção do refluxo9.

Neste trabalho o refluxo ocorreu em $28,5 \%$ dos animais no grupo da operação de Collis-Lind, mas apenas no teste com pressão sobre a parede gástrica, o que proporciona fator facilitador para a ocorrência do refluxo. Em situação normal o refluxo não ocorreu em nenhum dos animais. Portanto as técnicas se equivalem quanto à proteção contra o refluxo espontâneo. Os dados encontrados na literatura mostram a mesma observação seja em trabalhos experimentais ou clínicos 9,26,30. Oliveira, et al. ${ }^{20}$, realizaram fundoplicatura parcial (Lind) e total (Nissen) em coelhos e concluíram que ambos 
promovem aumento da pressão e comprimento da zona de alta pressão e seu aumento não depende do tipo de fundoplicatura realizada.

A avaliação da ocorrência do refluxo por aplicar pressão sobre o estômago foi muito utilizado nos trabalhos experimentais e todos mostraram o aumento da pressão intragástrica e no segmento de alongamento do esôfago envolvido pela válvula16,24,28.

No intraoperatório era observado a ocorrência do refluxo espontâneo ou com compressão do estômago através da coleta do líquido refluído no esôfago e análise com fita de pHmetria.

Porém, o estudo das pressões no tubo com a fundoplicatura era feito na peça cirúrgica ressecada. Isso foi usado por Butterfield ${ }^{4}$ em peças anatômicas retiradas de cadáver constituindo do esôfago distal, o estômago e o duodeno. Em seguida era colocado água no estômago com o duodeno fechado e observado refluxo no esôfago. Foram realizadas as operações de Nissen, Belsey, Hill e Thal-Hatafuku e mais uma vez observado a ocorrência do refluxo. Não ocorrendo refluxo espontâneo era aplicado pressão arbitrária de $250 \mathrm{mmHg}$ no estômago. Nesse trabalho as melhores técnicas foram a de Nissen e de Hill.

O fator mais importante considerado no controle do refluxo é a obtenção de zona de alta pressão junto do EIE e a sua extensão. Khan, et al. ${ }^{15}$, estudando cães, mostraram que os métodos cirúrgicos que colocam o EIE no abdome associado ao envolvimento pelo fundo gástrico, levam a aumento da zona de alta pressão e da sua extensão.

As variações de pressão no estômago e no neoesôfago com ou sem pressão sobre a parede gástrica não mostrou diferença significativa entre os dois grupos estudados aqui, mostrando respostas semelhantes. Nos trabalhos da literatura os que compararam as pressões entre as fundolicaturas parcial ou total, mostraram melhor resposta na fundoplicatura total ${ }^{9,22}$.

Quanto à extensão da zona de alta pressão também não houve diferença estatística entre os dois grupos estudados. Portanto, qualquer tipo de fundoplicatura é capaz de obter segmento suficiente para a contensão do refluxo. Ellis, et al. ${ }^{9}$, em trabalho experimental encontraram resultados também semelhantes na avaliação da extensão da zona de alta pressão na operação de Collis-Nissen e Collis-Belsey.

Como última informação, a operação de Collis associada à fundoplicatura parcial ou total, pode ser realizada por videocirurgia atraindo o benefício da cirurgia minimamente invasiva, obtendo em séries clínicas, resultados satisfatórios no controle do refluxo ${ }^{13,14,17,31,32}$. É, portanto, adequada para a contensão do refluxo gastroesofágico, podendo ser indicada para os casos graves de esôfago encurtado. Os trabalhos clínicos mostram que a técnica tem baixa incidência de recorrência dos sintomas e poucas falhas no controle do refluxo.

\section{CONCLUSÃO}

A operação de Collis-Lind ou Collis-Nissen impediu o refluxo gastroesofágico. As medidas das pressões e a extensão da zona de alta pressão foram semelhantes nos dois grupos estudados.

\section{AGRADECIMENTO}

Ao Prof Dr. Nelson Ary Brandalise que foi o orientador deste trabalho e continua a ensinar gerações de cirurgiões com sua capacidade, nos quais me incluo.

\section{REFERÊNCIAS}

1. Andriani AC, Neves TT. A gastroplastia em manga (sleeve gastrectomy) e o diabetes mellitus. ABCD Arq Bras Cir Dig . 2008; 21(3):133-135.

2. Agha FP, Trenhner SW, Orringer MB, Vinh PN. The combined Collis gastroplasty-Nissen fundoplication: surgical procedure and radiographic evaluation. AJR. 1985; 145:729-34.

3. Andreollo NA, Lopes LR, Coelho-Neto JS. Doença do refluxo gastroesofágico: qual a eficácia dos exames no diagnóstico? ABCD Arq Bras Cir Dig. 2010; 23(1): 6-10.

4. Butterfield WC. Current hiatal hernia repairs: similarities, mechanism and extended indications - an autopsy study. Surgery. 1971; 69:910-6.

5. Collis JL. An operation for hiatus hernia with short esophagus. J. Thorac. Surg. 1957; 34:768-78.

6. Collis JL. Surgical control of reflux in hiatus hernia. Am. J. Surg. 1968; 115:465-71.

7. Deitel M, Khanna RK, Hagen J, Ilves R. Vertical banded gastroplasty as an antireflux procedure. Am. J. Surg. 1988; 155:512-6.

8. Demos NJ. A simplified, improved technique for the Collis gastroplasty for dilatable strictures. Surg. Gynecol. Obst. 1976; 142:591-2.

9. Ellis FH, Leonardi HK, Dabuznsky L, Crozier RE. Surgery for short esophagus with stricture: an experimental and clinical manometric study. Ann. Surg. 1978; 188:341-50.

10. Gatzinsky P, Bergh NP. Hiatal hernia and shortened oesophagus. Acta Chir. Scand. 1979; 145:159-66.

11. Henderson RD, Boszko A, Mugashi F, Szcepanski MM, Marryatt G. Oesophageal replacement by a gastric tube: an experimental study of the properties of gastric tube. Br. J. Surg. 1974; 61:533-7.

12. Henderson RD. Reflux control following gastroplasty. Ann. Thorac. Surg. 1977; 24:206-14.

13. Jobe BA, Horvath KD, Swanstrom LL. Postoperative function following laparoscopic Collis gastroplasty for shortened esophagus. Arch. Surg., 133:867-74, 1998.

14. Johnson AB, Oddsdottir M, Hunter JG. Laparoscopic Collis gastroplasty and Nissen fundoplication: A new technique for the management of esophageal foreshortening. Surg. Endosc. 1998; 12:1055-60.

15. Khan TA, Crispin JS, Lind JF. Effect change of position on the function of the canine lower esophageal sphincter. Gastroenterol. 1974; 67:957-64.

16. Leonardi HK, Lee ME, El-Kurd MF, Ellis FH. An experimental study of the effectiveness of various antireflux operations. Ann. Thorac. Surg. 1977; 24:215-22.

17. Luketich JD, Grondin SC, Pearson FG. Minimally invasive approaches to acquired shortening of the esophagus: laparoscopic Collis-Nissen gastroplasty. Semin. Thorac. Cardiovasc. Surg. 2000; 12:173-8

18. Mattioli S, Lugaresi ML, Costantini M, Del Genio A, Di Martino N, Fei L, Fumagalli U, Maffettone $V$, Monaco L, Morino M, Rebecchi F, Rosati R, Rossi M, Santi S, Trapani V, Zaninotto G. The short esophagus: intraoperative assesment of esophageal length. J. 
Thorac. Cardiovasc. Surg, 2008; 136:834-41.

19. Mercer CD, Hill LD. Surgical management of peptic esophageal stricture. Twenty-year experience. J. Thorac. Cardiovasc. Surg. 1986; 91:371-8.

20. Oliveira WK, Henry MACA, Lerco MM. Manometric evaluation of lower esophageal sphincter in rabbits submitted to total and partial fundoplication. Acta Cir Bras. 2004;19(5): 555-564.

21. Orringer MB, Sloan $\mathrm{H}$. Collis-Belsey reconstruction of the esophagogastric junction. J. Thorac. Cardiovasc. Surg. 1976; 71:295-303.

22. Orringer MB, Sloan $\mathrm{H}$. Complcations and failings of the combined Collis-Belsey operation. J. Thorac. Cardiovasc. Surg. 1977; 74:726-35.

23. Pearson FG, Langer B, Henderson RD. Gastroplasty and Belsey hiatus repair - An operation for the management of peptic stricture with acquired short esophagus. J. Thorac. Cardiovasc. Surg. 1971; 61:50-63.

24. Pearson FG, Henderson RD. Experimental and clinical studies of gastroplasty in the management of acquired short esophagus. Surg. Gynecol. Obstet. 1973; 136:737-44.

25. Pearson FG, Henderson RD. Long-term follow-up of strictures managed by dilatation, modified Collis gastroplasty and Belsey hiatus hernia repair. Surgery. 1976; 80:396-404.

26. Pearson FG. Surgical management of acquired short esophagus with dilatable peptic stricture. World J. Surg. 1977; 1:463-73.

27. Pearson FG, Cooper JD, Patterson GA, Ramires J, Todd TR. Gastroplasty and fundoplication for complex problems - Long- term results. Ann. Surg. 1987; 206:473-81.

28. Sicular A, Cohen B, Zimmerman A, Kark AE. The significance of on intra-abdominal segment of canine esophagus as a competent anti-reflux mechanism. Surgery. 1967; 61:784-90.

29. Steichen FM. Abdominal approach to the Collis gastroplasty and Nissen fundoplication. Surg. Gynecol. Obstet. 1986; 162:273-5.

30. Stirling MC, Orringer MB. The combined Collis-Nissen operation for esophageal reflux strictures. Ann. Thorac. Surg. 1988; 45:148-57.

31. Swanstrom LL, Marcus DR, Galloway GQ. Laparoscopic Collis gastroplasty is the treatment of choice for the shortened esophagus. Am. J. Surg. 1996; 171:477-81.

32. Terry ML, Vernon A, Hunter JG. Stapled-wedge Collis gastroplasty for the shortened esophagus. Am. J. Surg. 2004; 188:195-9.

33. Thomas-Ridocci M, Paris F, Carbonell-Antoli C, Mora F, Molina R, Padilha J, Carbonell-Canti C, Moreno-Osset E, Guijarro R, Benages A. Total fundoplication with or without gastroplasty for gastroesphageal reflux: comparative study. Ann. Thorac. Surg. 1985; 39:508-11.

34. Valezi AC, Mali-Junior J, Brito EM, Marson AC. Gastroplastia vertical com bandagem em y-de-roux: análise de resultados. Rev Col Bras Cir. 2004; 31(1):49-56. 